\title{
Erratum to: Mechano-Transduction Signals Derived from Self-Assembling Peptide Nanofibers Containing Long Motif of Laminin Influence Neurogenesis in In-Vitro and In-Vivo
}

\author{
Shima Tavakol ${ }^{1,2,3} \cdot$ Sayed Mostafa Modarres Mousavi ${ }^{4} \cdot$ Behnaz Tavakol $^{5}$. \\ Elham Hoveizi $^{6}$ - Jafar Ai ${ }^{7,8}$ - Seyed Mahdi Rezayat Sorkhabadi ${ }^{2,9,10,11}$
}

Published online: 1 September 2016

(C) Springer Science+Business Media New York 2016

\section{Erratum to: Mol Neurobiol}

DOI 10.1007/s12035-016-9836-Z

The original paper of this article unfortunately contains error.

The correct first affiliation of Dr. Shima Tavakol should be written as "Cellular and Molecular Research Center, Iran University of Medical Sciences, Tehran, Iran." instead of "Drug Nanocarriers Research Core, Razi Drug Research Center, Iran University of Medical Sciences, Tehran, Iran”.

This change is requested by the author and hereby published.

The online version of the original article can be found at http://dx.doi.org/ 10.1007/s12035-016-0006-0.

Shima Tavakol

shima.tavakol@yahoo.com

Jafar Ai

Jafar_ai@tums.ac.ir

Seyed Mahdi Rezayat Sorkhabadi

rezayat@tums.ac.ir

1 Cellular and Molecular Research Center, Iran University of Medical Sciences, Tehran, Iran

2 Department of Medical Nanotechnology, School of Advanced Technologies in Medicine, Tehran University of Medical Sciences, Tehran 1417755469, Iran

3 Student's Scientific Research Center, Tehran University of Medical Sciences, Tehran, Iran

4 Shefa Neuroscience Research Center, Khatam Alanbia Hospital, Tehran, Iran
5 School of Medicine, Kashan University of Medical Sciences, Kashan, Iran

6 Department of Biology, Faculty of Sciences, Shahid Chamran University of Ahvaz, Ahvaz, Iran

7 Department of Tissue Engineering, School of Advanced Technologies in Medicine, Tehran University of Medical Sciences, Tehran, Iran

8 Brain and Spinal Injury Research Center, Imam Hospital, Tehran University of Medical Sciences, Tehran 1417755469, Iran

9 Department of Toxicology and Pharmacology, School of Pharmacy, Pharmaceutical Sciences Branch, Islamic Azad University (IAUPS), Tehran, Iran

10 Experimental Medicine Research Center, Tehran University of Medical Sciences, Tehran, Iran

11 Department of Pharmacology, School of Medicine, Tehran University of Medical Sciences, Tehran, Iran 